$10-2006$

\title{
An Initial Non-Equilibrium Porous-Media Model for CFD Simulation of Stirling Regenerators
}

\author{
Roy C. Tew \\ NASA Glenn Research Center \\ Terry Simon \\ University of Minnesota \\ David Gedeon \\ Gedeon Associates
}

Mounir B. Ibrahim

Cleveland State University, m.ibrahim@csuohio.edu

Wei Rong

Cleveland State University

Follow this and additional works at: https://engagedscholarship.csuohio.edu/enme_facpub

Part of the Mechanical Engineering Commons

How does access to this work benefit you? Let us know!

\section{Recommended Citation}

Tew, Roy C.; Simon, Terry; Gedeon, David; Ibrahim, Mounir B.; and Rong, Wei, "An Initial Non-Equilibrium Porous-Media Model for CFD Simulation of Stirling Regenerators" (2006). Mechanical Engineering Faculty Publications. 270.

https://engagedscholarship.csuohio.edu/enme_facpub/270

This Report is brought to you for free and open access by the Mechanical Engineering Department at EngagedScholarship@CSU. It has been accepted for inclusion in Mechanical Engineering Faculty Publications by an authorized administrator of EngagedScholarship@CSU. For more information, please contact library.es@csuohio.edu. 
NASA/TM-2006-214391

(245)

\section{An Initial Non-Equilibrium Porous-Media Model for CFD Simulation of Stirling Regenerators}

Roy C. Tew

Glenn Research Center, Cleveland, Ohio

Terry Simon

University of Minnesota, Minneapolis, Minnesota

David Gedeon

Gedeon Associates, Athens, Ohio

Mounir Ibrahim and Wei Rong

Cleveland State University, Cleveland, Ohio 


\section{NASA STI Program . . . in Profile}

Since its founding, NASA has been dedicated to the advancement of aeronautics and space science. The NASA Scientific and Technical Information (STI) program plays a key part in helping NASA maintain this important role.

The NASA STI Program operates under the auspices of the Agency Chief Information Officer. It collects, organizes, provides for archiving, and disseminates NASA's STI. The NASA STI program provides access to the NASA Aeronautics and Space Database and its public interface, the NASA Technical Reports Server, thus providing one of the largest collections of aeronautical and space science STI in the world. Results are published in both non-NASA channels and by NASA in the NASA STI Report Series, which includes the following report types:

- TECHNICAL PUBLICATION. Reports of completed research or a major significant phase of research that present the results of NASA programs and include extensive data or theoretical analysis. Includes compilations of significant scientific and technical data and information deemed to be of continuing reference value. NASA counterpart of peer-reviewed formal professional papers but has less stringent limitations on manuscript length and extent of graphic presentations.

- TECHNICAL MEMORANDUM. Scientific and technical findings that are preliminary or of specialized interest, e.g., quick release reports, working papers, and bibliographies that contain minimal annotation. Does not contain extensive analysis.

- CONTRACTOR REPORT. Scientific and technical findings by NASA-sponsored contractors and grantees.
- CONFERENCE PUBLICATION. Collected papers from scientific and technical conferences, symposia, seminars, or other meetings sponsored or cosponsored by NASA.

- SPECIAL PUBLICATION. Scientific, technical, or historical information from NASA programs, projects, and missions, often concerned with subjects having substantial public interest.

- TECHNICAL TRANSLATION. Englishlanguage translations of foreign scientific and technical material pertinent to NASA's mission.

Specialized services also include creating custom thesauri, building customized databases, organizing and publishing research results.

For more information about the NASA STI program, see the following:

- Access the NASA STI program home page at http://www.sti.nasa.gov

- E-mail your question via the Internet to help@sti.nasa.gov

- Fax your question to the NASA STI Help Desk at 301-621-0134

- Telephone the NASA STI Help Desk at 301-621-0390

- Write to:

NASA STI Help Desk

NASA Center for AeroSpace Information 7121 Standard Drive Hanover, MD 21076-1320 
NASA/TM-2006-214391

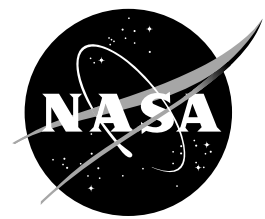

\section{An Initial Non-Equilibrium Porous-Media Model for CFD Simulation of Stirling Regenerators}

Roy C. Tew

Glenn Research Center, Cleveland, Ohio

Terry Simon

University of Minnesota, Minneapolis, Minnesota

David Gedeon

Gedeon Associates, Athens, Ohio

Mounir Ibrahim and Wei Rong

Cleveland State University, Cleveland, Ohio

Prepared for the

Fourth International Energy Conversion Engineering Conference and Exhibit (IECEC) sponsored by the American Institute of Aeronautics and Astronautics San Diego, California, June 26-29, 2006

National Aeronautics and

Space Administration

Glenn Research Center

Cleveland, Ohio 44135 


\section{Acknowledgments}

The work described in this paper was performed for the NASA Science Mission Directorate (SMD) and the Radioisotope Power System (RPS) Program.

Level of Review: This material has been technically reviewed by technical management.

Available from

NASA Center for Aerospace Information

7121 Standard Drive

Hanover, MD 21076-1320
National Technical Information Service 5285 Port Royal Road Springfield, VA 22161

Available electronically at http://gltrs.grc.nasa.gov 


\title{
An Initial Non-Equilibrium Porous-Media Model for CFD Simulation of Stirling Regenerators
}

\author{
Roy C. Tew \\ National Aeronautics and Space Administration \\ Glenn Research Center \\ Cleveland, Ohio 44135 \\ Terry Simon \\ University of Minnesota \\ Minneapolis, Minnesota 55455 \\ David Gedeon \\ Gedeon Associates \\ Athens, Ohio 45701 \\ Mounir Ibrahim and Wei Rong \\ Cleveland State University \\ Cleveland, Ohio 44115
}

\begin{abstract}
The objective of this paper is to define empirical parameters (or closure models) for an initial thermal non-equilibrium porous-media model for use in Computational Fluid Dynamics (CFD) codes for simulation of Stirling regenerators. The two CFD codes currently being used at Glenn Research Center (GRC) for Stirling engine modeling are Fluent and CFD-ACE. The porous-media models available in each of these codes are equilibrium models, which assume that the solid matrix and the fluid are in thermal equilibrium at each spatial location within the porous medium. This is believed to be a poor assumption for the oscillating-flow environment within Stirling regenerators; Stirling 1-D regenerator models, used in Stirling design, use non-equilibrium regenerator models and suggest regenerator matrix and gas average temperatures can differ by several degrees at a given axial location and time during the cycle. A NASA regenerator research grant has been providing experimental and computational results to support definition of various empirical coefficients needed in defining a non-equilibrium, macroscopic, porous-media model (i.e., to define "closure" relations). The grant effort is being led by Cleveland State University, with subcontractor assistance from the University of Minnesota, Gedeon Associates, and Sunpower, Inc. Friction-factor and heat-transfer correlations based on data taken with the NASA/Sunpower oscillating-flow test rig also provide experimentally based correlations that are useful in defining parameters for the porous-media model; these correlations are documented in Gedeon Associates' Sage Stirling-Code Manuals. These sources of experimentally based information were used to define the following terms and parameters needed in the non-equilibrium porous-media model: hydrodynamic dispersion, permeability, inertial coefficient, fluid effective thermal conductivity (including thermal dispersion and estimate of tortuosity effects), and fluid-solid heat transfer coefficient. Solid effective thermal conductivity (including the effect of tortuosity) was also estimated. Determination of the porous-media model parameters was based on planned use in a CFD model of Infinia's Stirling Technology Demonstration Convertor (TDC), which uses a random-fiber regenerator matrix. The non-equilibrium porous-media model presented is considered to be an initial, or "draft," model for possible incorporation in commercial CFD codes, with the expectation that the empirical parameters will likely need to be updated once resulting Stirling CFD model regenerator and engine results have been analyzed. The emphasis of the paper is on use of available data to define empirical parameters (and closure models)
\end{abstract}


needed in a thermal non-equilibrium porous-media model for Stirling regenerator simulation. Such a model has not yet been implemented by the authors or their associates. However, it is anticipated that a thermal non-equilibrium model such as that presented here, when incorporated in the CFD codes, will improve our ability to accurately model Stirling regenerators with CFD relative to current thermal-equilibrium porous-media models.

\section{Nomenclature}



Greek Symbols

$\beta=$ porosity

$\rho=\operatorname{density}\left(\mathrm{kg} / \mathrm{m}^{3}\right)$

$\mu=$ absolute viscosity $(\mathrm{kg} / \mathrm{m}-\mathrm{s})$

$v=$ dynamic viscosity $\left(\mathrm{m}^{2} / \mathrm{s}\right)$

\section{Introduction}

The Advanced Stirling Convertor (ASC) is being developed as a light-weight, high-efficiency Advanced Stirling Radioisotope Generator (SRG) for use on potential NASA Space Science missions. Potential missions include providing spacecraft onboard-electric power for deep-space missions or power for lunar applications, or un-piloted Mars rovers. Advanced Stirling convertors would provide substantial performance and mass benefits for these missions and could also allow the use of Stirling radioisotope power systems for radioisotope electric propulsion. A combined Stirling convertor/cooler based on a high temperature heater head may enable an extended-duration Venus surface mission. Therefore, GRC is also developing advanced technology for Stirling convertors, aimed at improving specific power and efficiency of the convertor and the overall power system, and confirming and enhancing reliability. Performance and mass improvement goals have been established for these next generation 
Stirling radioisotope power systems. Efforts are underway to achieve these goals, both in-house at GRC and via grants and contracts. These efforts include use of multi-dimensional (multi-D) Stirling computational-fluiddynamics (CFD) models to enhance knowledge of component performance details and reliability, high-temperature materials, advanced controllers, low-vibration techniques, advanced regenerators, and a lightweight convertor., 2

The objective of this paper is to report on definition of empirical parameters for an initial thermal nonequilibrium porous-media model intended for use in multi-D Stirling codes $^{3-5}$ for simulation of regenerators. Experimental data from a regenerator research grant ${ }^{6-8}$ and experimentally based correlations derived from oscillating-flow rig test data ${ }^{9}$ were used in defining parameters needed in the non-equilibrium porous-media model. The grant is led by Cleveland State University (CSU) with subcontractor assistance from the University of Minnesota (UMN), Gedeon Associates and Sunpower Inc. Determination of the particular porous media model parameters presented was based on planned use in a CFD model of Infinia's Stirling Technology Demonstration Convertor (TDC). The thermal non-equilibrium porous-media model presented is considered to be an initial, or "draft," model for possible incorporation in commercial CFD codes (which now contain thermal equilibrium porousmedia models), with the expectation that empirical parameters or heuristic terms in the equations may need to be updated once resulting Stirling CFD model regenerator and engine results have been analyzed. The emphasis of the paper is on use of available data to define the empirical parameters (or closure models) needed in non-equilibrium porous-media models for Stirling regenerator simulation. Such a porous-media model has not yet been implemented in a Stirling CFD model by the authors or their associates.

Two CFD codes, Fluent ${ }^{\dagger \dagger}$ and CFD-ACE ${ }^{\ddagger}$, have been used at Glenn Research Center (GRC) for modeling of Infinia's Stirling TDC. The porous-media models available in each of these codes are thermal equilibrium models, which assume that the solid matrix and the fluid are in local thermal equilibrium (thus at the same temperature) at each spatial interface between them within the porous medium. This is believed to be a poor assumption for the oscillating-flow environment within Stirling regenerators; some results supporting this belief are: (1) Experimental measurements in a large-scale replica of such a regenerator show solid and fluid temperatures differ by as much as 2 degrees $\mathrm{C}$ over an engine cycle, as shown in Fig. ${ }^{\S \S}$. Characteristics of this large-scale replica and the tests were: $90 \%$ porosity, stainless steel 304 welded screens, 200 layers of $6.3 \mathrm{~mm}$ x $6.3 \mathrm{~mm}$ mesh, $0.81 \mathrm{~mm}$ wire diameter; test section dimensionless parameters were similar to those of the TDC (the temperature measurements were made with

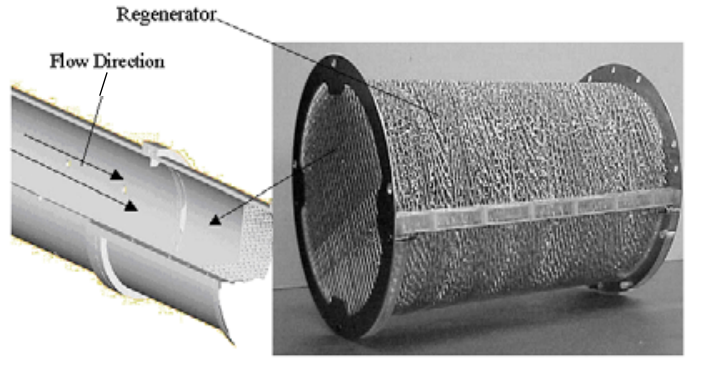

Figure 1. Wire-Screen Regenerator (Considered to Approximate Random Fiber) and Test Results. The Temperature Measurements, shown at right, were made with a Hot Wire at the Axial Center of the $80^{\text {th }}$ Layer of Screen (from the Cold End).



hot-wire anemometry). (2) This same experimental data is shown in Figure 2, along with (a) a "microscopic" CFD simulation of the non-equilibrium temperatures of one layer of the test rig welded screen and adjacent fluid ${ }^{10}$, and (b) a CFD-ACE "macroscopic" equilibrium porous-media model simulation of the "lumped" solid plus fluid temperature over a cycle. Note that the variation of the calculated macroscopic thermal-equilibrium-model lumped solid/fluid temperature over the cycle (lying between the CFD microscopic and the measured solid temperatures in Fig. 2), is almost the same as that of the non-equilibrium solid temperature variations-due to the much greater specific heat of the solid. (3) Fig. 3 shows Sage 1-D TDC model predictions of the regenerator solid and fluid

\footnotetext{
${ }^{\dagger \dagger}$ Internet website www.fluent.com or do internet search on "Fluent"

㧊 Internet website www.esi-group.com/SimulationSoftware/CFD-ACE/ or do internet search on "CFD-ACE”

$\S \S$ Simon, T., “Contributions to Regenerator Modeling,” Presentation for Regenerator Modeling Panel Discussion, $2^{\text {nd }}$ International Energy Conversion Engineering Conference, Providence, RI, August 18, 2004.
} 


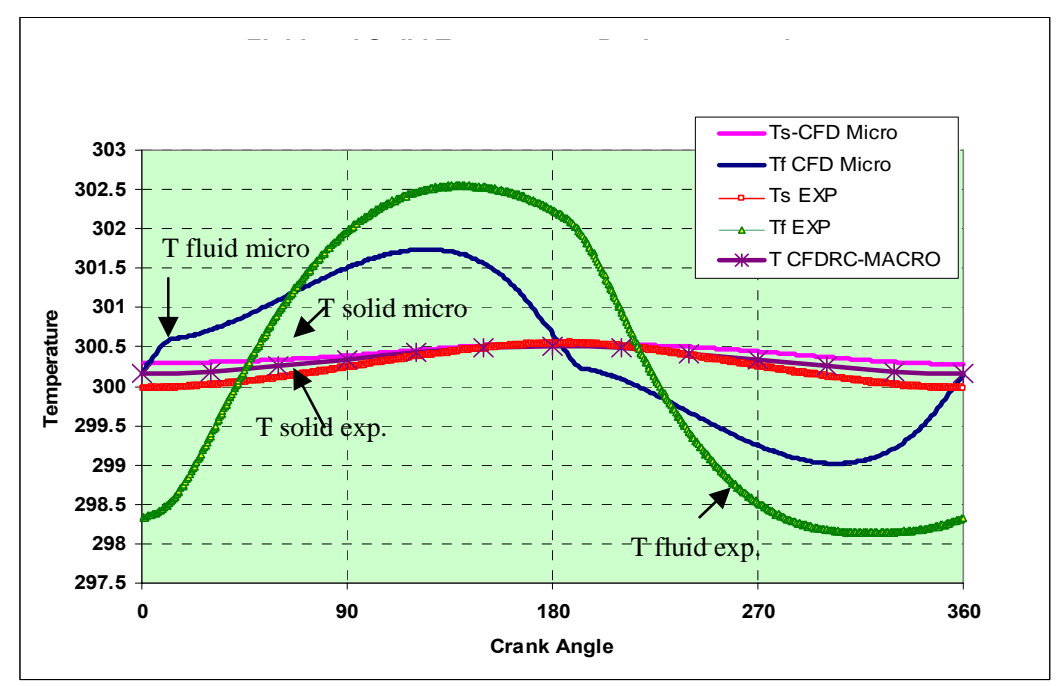

Figure 2. CFD Microscopic Calculations of Solid and Fluid Temperatures, and Macroscopic Equilibrium Solid+Fluid Temperature, deg. K--superimposed on the UMN Experimental Temperatures from Fig. 1

matrix/fluid thermal equilibrium would be inappropriate for the Stirling TDC regenerator. temperatures $^{* * *}$. Differences in solid and fluid temperatures range up 20 degrees $K$ at the ends of the regenerator and $\sim 1-2$ degrees at the center of the regenerator. These calculations, made by the first author at GRC, yielded an enthalpy flux loss over the cycle of about $22 \mathrm{~W}$, or approximately $8 \%$ of the predicted 288 $\mathrm{W}$ of heat into the hot-end of the convertor (predicted electrical power was about $56 \mathrm{~W}$ at $\sim 83 \mathrm{~Hz}$ frequency); one would expect that neglect of the temperature difference between the regenerator solid and fluid, and the consequent entropy generation, would produce underestimation of regenerator enthalpy-flux losses. Therefore these results, both measurements and CFD simulations, suggest the assumption of solid-
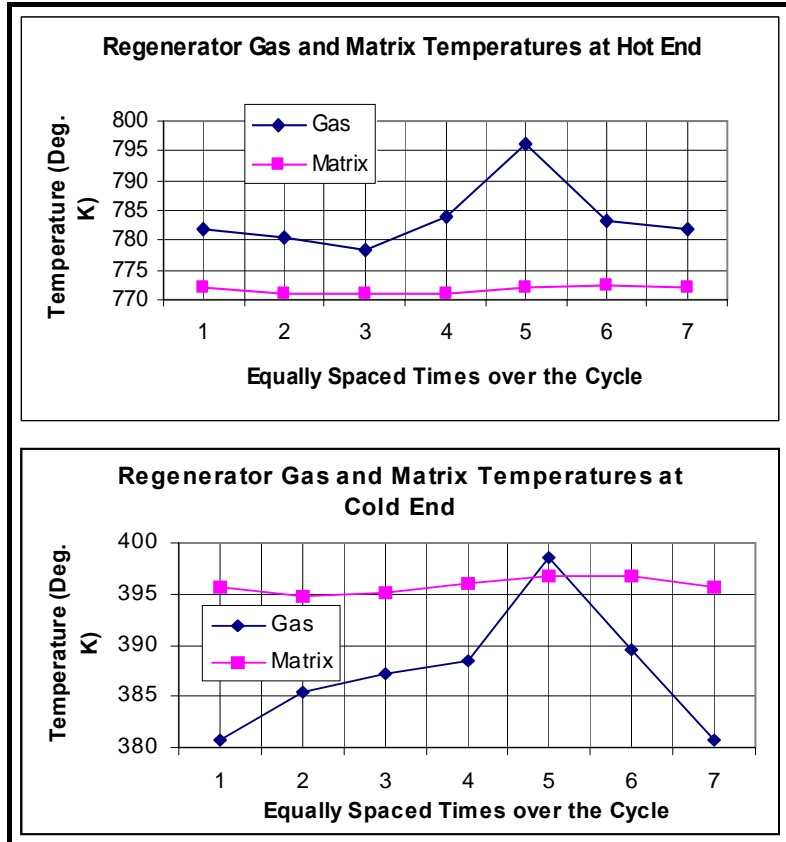



TDC Operating Conditions \& Predictions

- Operating Conditions (Not Design) were

- $\quad$ Hot-End Temperature $=823 \mathrm{~K}(550 \mathrm{C})$

- $\quad$ Cold-End Temperature = $363 \mathrm{~K}(90 \mathrm{C})$

- $\quad$ Frequency of Operation $=82.5 \mathrm{~Hz}$

- Helium Mean Pressure = 2.59 MPa (376 psi)

- Performance Predictions were

Engine Electrical Power $=55.9 \mathrm{We}$

Engine Efficiency $=19.4 \%$

Heat Into Engine $=\mathbf{2 8 8} \mathrm{W}$

Regenerator Enthalpy Flux Loss $=22.4 \mathrm{~W}$

Regen. Enthalpy Flux Loss $\equiv \oint \dot{m} c_{p} T_{g a s} d t$

Figure 3. Sage 1-D Thermal Non-Equilibrium Regenerator Model, Fluid \& Solid Temperature Predictions for TDC

A survey of the porous-media literature supports the need for thermal non-equilibrium porous-media models for thermal storage applications, as in Stirling regenerators. In Singh ${ }^{11}$ it is noted that in a thermal storage system, heat transfer between the solid and fluid phases is a key phenomenon and both the phases are inherently in thermal nonequilibrium. Hence the corresponding models of heat transfer will necessarily be of the 2-energy-equation type, one equation each for the fluid and solid phase temperature. Results are shown in the paper of 1- and 2-energy-equation temperature calculations (i.e. using thermal- equilbrium and non-equilibrium models) for steady-flow through

*** Tew, R., “Overview of Stirling Regenerator (Porous Media) CFD Modeling Problems,” Presentation for Workshop on Convective Heat Transfer in Porous Media, Hosted by NASA Glenn Research Center, North Olmsted, OH, April 28-29, 2004. 
porous-media with thermal fields which evolve with time; 1- and 2-energy-equation temperature profiles are compared for glass/water and metal/air porous-media systems over ranges of Reynolds number and porous-media flow lengths.

Amiri and Vafai ${ }^{12}$ examined the validity of the thermal-equilibrium assumption via a one-dimensional study of incompressible flow through a packed bed. They noted that the transient response of the solid structure is crucial to the performance of packed beds when used for thermal storage applications, and the solid-to-fluid heat transfer coefficient is a major resistance to heat transfer at the interface between the fluid and the solid matrix. The "microscopic" governing equations were volume averaged over a representative elementary volume to arrive at the "macroscopic" transport equations, including separate fluid and solid phases; the resulting 1-D, incompressible, volume-averaged macroscopic equations used by Amiri and Vafai are a reduced form of the more general macroscopic equations given below in this paper. Temperature differences between fluid and solid were calculated for a range of Reynolds No.'s and Darcy No.'s (dimensionless permeability). It was found that the ratio of fluid-tosolid thermal conductivity was an important parameter in determining local temperature difference between fluid and solid, for a given set of operating conditions. This analysis was also for steady flow through the porous media (packed beds) with developing thermal fields.

Minkowycz ${ }^{13}$ analyzes departure from local thermal equilibrium in porous media due to a rapidly changing heat source. The analysis is done for a sinusoidally-varying imposed heat-flux at one of the boundaries for the cases of no-flow and steady-flow in the same direction as the imposed heat-flux. It is noted that local thermal equilibrium is an often-used hypothesis when studying heat transfer in porous media, but examination of non-equilibirum phenomena show that this hypothesis is usually not valid during rapid heating and cooling. Oscillatory plots of fluid and solid dimensionless temperatures as a function of dimensionless time over the cycle are shown for various combinations of dimensionless frequency, fluid and solid heat capacities, and dimensionless "Sparrow No.'s;" Sparrow No. is defined to be a product of one form of the more familiar Biot number and a dimensionless ratio of lengths. It is noted that in the presence of rapid surface heat input variations, local thermal equilibrium fails to exist and large errors can be realized, depending on the value of the thermophysical properties; and the value of the Sparrow number appears to be indicative of the presence, or not, of local thermal equilibrium for applications dealing with rapidly changing heat inputs.

Muralidhar $^{14}$ analyzed the effect of pulsating flow of gas and the accompanying heat transfer within a regenerator made from wire-mesh screens, of the type used in Stirling cycle devices. A harmonic analysis was used to examine fully-developed but unsteady gas flow in a regenerator assuming a non-Darcy, thermally nonequilibrium porous medium. It is noted that one assumption often adopted in the published literature is the thermal equilibrium between fluid in the pores and the solid material of the porous medium; however, thermal performance of regenerators totally depends on heat transfer between fluid and solid phases. Therefore, the individual phases should have a local temperature difference from one to the other. Unfortunately, although temperature profiles and regenerator effectiveness are explored as functions of Reynolds No., pulsating frequency, thermal properties of gas and solid, and the mesh structure - the local temperature differences between gas and solid do not appear to be given in the paper.

Gedeon ${ }^{15}$ developed a 2-D CFD "Manifest" (for "manifold estimate”) computer code for simulation of Stirling regenerators and their manifolds (that was capable of extension to other Stirling components). In the process of developing this code, he derived a non-equilibrium macroscopic porous-media model, starting from the generalized Navier-Stokes equations. These integral-form porous-media equations were also converted to curvilinear form for use in Manifest. Gedeon used Manifest to study jetting into the porous matrix (as from adjacent heat exchangers) and the effect of side-inlet regenerator manifolds. Side-inlet manifolds appeared to greatly enhance the possibility that flow through the regenerator would be non-uniform and thus decrease regenerator performance. The form of Gedeon's integral-form non-equlibrium porous-media equations differs from the differential form shown in this paper.

Thermal equilibrium porous-media models, or 1-energy-equation models, are consistent with the assumption of infinite heat-transfer coefficients between fluid and solid phases and completely neglect entropy generation due to the radial heat transfer between the fluid and solid phases (since the local temperature difference between the phases is zero). However, unless the solid heat capacity of the porous-medium is essentially infinite relative to that of the fluid, thermal-equilibrium-model hot-end and cold-end regenerator fluid exit temperatures can still vary over the cycle; so, in general, thermal equilibrium porous-media models of Stirling regenerators will still yield non-zero regenerator enthalpy flux losses over the cycle, though one would expect that these losses would be underestimated. 


\section{A Compressible-Flow Non-Equilibrium Porous-Media Model for CFD Codes}

Initial values of parameters needed for a macroscopic non-equilibrium porous-media model are defined below for use in modeling Technology Demonstration Convertor (TDC) regenerators in CFD codes. Fluid continuity, momentum and energy equations, and a solid-energy equation, are stated for reference in defining the parameters needed. Experimental and computational data generated under a regenerator research grant ${ }^{6-8}$, and data from an oscillating-flow test rig ${ }^{9}$ are used in defining this initial set of parameters (i.e., in defining closure models).

First define superficial (Darcian), approach, velocity as $U=\langle u\rangle=u a / a_{\text {total }}=u \beta$. This is also the volume-flow rate through a unit cross-sectional area of the solid-plus-fluid. It is determined by averaging the velocity over a region that is small with respect to the macroscopic flow dimension but large with respect to the matrix-flowchannel size. In the above equation for superficial velocity, " $a$ ” is the average fluid-flow area within the total solidplus-fluid cross-sectional area, $a_{\text {total }}$, that the velocity is averaged over to determine the superficial velocity, $U$. The porosity is $\beta$. The average-flow-channel fluid velocity (or local porous-media velocity) is $u$, corresponding to the superficial velocity, $U=\langle u\rangle$, and $u=\langle u\rangle / \beta$.

\section{A. Non-Equilibrium Porous-Media Conservation Equations}

The incompressible-flow, non-equilibrium, porous-media equations from Ibrahim and Rong ${ }^{10}$ were used as a starting point; they used the volume-averaging approach described in Whitaker ${ }^{16}$ in deriving their macroscopic porous-media equations. Rewritten for compressible flow ${ }^{26}$, and including a hydrodynamic dispersion term from Ayyaswamy $^{\text {t+t }}$, the fluid continuity, momentum, and energy equations, respectively, written in terms of the superficial, or approach, or Darcian velocity are (where the brackets, $<>$, denote volume averaging):

$$
\begin{gathered}
\frac{\partial\langle\rho\rangle^{f}}{\partial t}+\frac{1}{\beta} \nabla \cdot\left[\langle\rho\rangle^{f}\langle\mathbf{u}\rangle\right]=0 \\
\frac{1}{\beta} \frac{\partial\left(\langle\rho\rangle^{f}\langle\mathbf{u}\rangle\right)}{\partial t}+\frac{1}{\beta^{2}} \nabla \cdot\left[\langle\rho\rangle^{f}\langle\mathbf{u}\rangle\langle\mathbf{u}\rangle\right]=-\nabla\langle p\rangle^{f}+\nabla \cdot\left(\frac{\left\langle v_{\text {eff }}\right\rangle^{f}}{\beta}\langle\rho\rangle^{f} \nabla\langle\mathbf{u}\rangle-\frac{\langle\rho\rangle^{f}}{\beta}\langle\tilde{\mathbf{u}} \tilde{\mathbf{u}}\rangle\right) \\
-\frac{\langle\mu\rangle^{f}}{K}\langle\mathbf{u}\rangle-\langle\rho\rangle^{f} \frac{{ }^{C} f}{\sqrt{K}}|\langle\mathbf{u}\rangle|\langle\mathbf{u}\rangle \\
\frac{\partial\left(\langle\rho\rangle^{f}\langle h\rangle^{f}\right)}{\partial t}+\frac{1}{\beta} \nabla \cdot\left[\langle\rho\rangle^{f}\langle\mathbf{u}\rangle\langle h\rangle^{f}\right]=\nabla \cdot\left[\overline{\bar{k}}_{f e} \bullet \nabla\langle T\rangle^{f}\right]+\left(\frac{\mu}{K}+\langle\rho\rangle^{f} \frac{C_{f}}{\sqrt{K}}|\mathbf{u}|\right) \mathbf{u} \cdot \mathbf{u}+ \\
h_{s f} \frac{d A_{s f}}{d V_{f}}\left(\langle T\rangle^{s}-\langle T\rangle^{f}\right)+\frac{d\langle p\rangle^{f}}{d t}
\end{gathered}
$$

where the wetted area per unit fluid volume, $\frac{d A_{s f}}{d V_{f}}=\frac{1}{r_{h}} \quad$ (and $r_{h}=$ hydraulic radius) and $\frac{d\langle p\rangle^{f}}{d t}$ is a substantial or material derivative. Finally, the solid energy equation is:

t+† Ayyaswamy, P. S., University of Pennsylvania, Private Communication, 2004. Ayyaswamy described this ũu term as representing "the additional stresses due to the correlation between the velocity fluctuations associated with the hydrodynamic dispersion due to the solid matrix.” A closure model for the term is discussed later in this paper.

\# The effective viscosity used in the "Brinkman term" of this momentum equation is different than the molecular viscosity, in general, as a result of the solid/fluid interfaces within the matrix. The Brinkman term computes momentum transport where there are velocity gradients within the flow. Examples would be the jetting of fluid from adjacent heat exchanger passages into the matrix, etc.

$\S \S$ The pressure-work term, $\frac{d\langle p\rangle^{f}}{d t}$, is the form for an ideal gas (helium, here). In general it should be written $\beta_{c t e} T \frac{d\langle p\rangle^{f}}{d t}$. For an ideal gas, the coefficient of thermal expansion, $\beta_{c t e}=\frac{1}{T}$.

${ }^{* * * *}$ Burmeister ${ }^{17}$ suggests the form of the fluid energy viscous dissipation terms, $\left(\frac{\mu}{K}+\langle\rho\rangle^{f} \frac{C_{f}}{\sqrt{K}}|\mathbf{u}|\right) \mathbf{u} \cdot \mathbf{u}$, here-which is consistent with the Darcy-Forchheimer terms in the momentum equation, (2). 


$$
\frac{\partial\left(\rho_{s} C_{s}\langle T\rangle^{s}\right)}{\partial t}=\nabla \cdot\left[\overline{\bar{k}}_{s e} \bullet \nabla\langle T\rangle^{s}\right]-h_{s f} \frac{d A_{s f}}{d V_{s}}\left(\langle T\rangle^{s}-\langle T\rangle^{f}\right)
$$

where the wetted area per unit solid volume, $\frac{d A_{s f}}{d V_{s}}=\frac{1}{r_{h}} \frac{1}{1-\beta}$.

Kaviany ${ }^{18}$ has described a momentum equation very similar to equation (2) as a "semiheuristic" equation. That is, most of the terms are multi-dimensional terms based on derivation from fundamentals. However, the last two terms of equation (2) are based on a "heuristic" extension from a 1D momentum equation (i.e., this is a "speculative extension" of an experimentally based 1D equation to serve as a guide in study of multi-dimensional problems, that has not been experimentally verified for multi-dimensional problems). Thus, in this initial or "draft" nonequilibrium porous-media model, we will assume that permeability is isotropic (Even though we know that the wirescreen used in engines and the UMN experiments is not isotropic, and because of the method of fabrication of random fiber material used in modern engines, it is believed that most of the lengths of those fibers, also, lie in planes perpendicular to the flow direction). Thus, as this porous-media model is improved upon, it may be desirable in the future to use anisotropic permeability. There are forms of Equation (2) with terms similar to the last two in equation (2), but in which the permeability is a tensor quantity ${ }^{16}$. But, in equation (2), permeability is a scalar quantity assumed to be the same for all directions. Similar statements can be made for the inertial term multiplier of equation (2), i.e. $C_{f} / \sqrt{K}$.

The expressions or parameters in the above equations needing definition, for use in modeling Stirling regenerators, are: (1) the hydrodynamic dispersion term in the momentum equation; (2) scalar permeability, $K$, and (3) scalar inertial coefficient, $C_{f}$, in the momentum equation; (4) the fluid effective thermal conductivity, $\overline{\bar{k}}_{f e}$, in the fluid-energy equation; (5) the solid effective thermal conductivity, $\overline{\bar{k}}_{s e}$, in the solid-energy equation, and (6) the heat-transfer coefficient between fluid and solid, $h_{s f}$, appearing in both fluid and solid energy equations . Note that the thermal conductivity expressions in the energy equations are tensors, though they are expected to have nonzero values only on the diagonals. The products of velocity vectors in the momentum equation are also tensors.

\section{Hydrodynamic Dispersion}

In the fluid momentum equation, equation (2) above, there is a hydrodynamic dispersion term:

$$
\frac{1}{\beta}\langle\tilde{\mathbf{u}} \tilde{\mathbf{u}}\rangle \cong \tilde{\mathbf{u}} \tilde{\mathbf{u}}
$$

where $\beta$ is porosity; $\mathbf{u}$ is the average-channel fluid, or local, velocity inside the matrix, and $\tilde{\mathbf{u}}$ is the spatial-variation of the average-channel fluid velocity inside the matrix. $\tilde{\mathbf{u}} \tilde{\mathbf{u}}$ is a tensor quantity which can be expanded in the form:

$$
\tilde{\mathbf{u}} \tilde{\mathbf{u}}=\mathbf{i i} \tilde{u} \tilde{u}+\mathbf{i} \mathbf{j} \tilde{u} \tilde{v}+\mathbf{i k} \tilde{u} \tilde{w}+\mathbf{j i} \tilde{v} \tilde{u}+j \mathbf{j} \tilde{v} \tilde{v}+\mathbf{j} \mathbf{k} \tilde{v} \tilde{w}+\mathbf{k i} \tilde{w} \tilde{u}+\mathbf{k} \mathbf{j} \tilde{w} \tilde{v}+\mathbf{k} \mathbf{k} \tilde{w} \tilde{w}
$$

For transport normal to the principal flow direction which is assumed to be the axial, $\mathbf{i}$, direction, the mean velocity gradient is in the radial, $\mathbf{j}$, direction and the term of interest (i.e. of significance) is $\tilde{u} \tilde{v}$, where $v$ is the velocity component in that direction, $=|\mathbf{u}| \mathbf{j}$. Therefore the term from the above expression for transport normal to the flow is $|\tilde{u} \tilde{v}|$ or $|\tilde{v} \tilde{u}|$. The dispersion represented by this term is of axial momentum, $\rho u$, but this term is in the direction normal to $u$ (in the direction of $v$ ).

In $\mathrm{Niu}^{7}$ it was argued that this hydrodynamic dispersion could be equated to turbulent shear stress when the dispersion of interest is not in the direction of the mean flow, or $\overline{\langle\tilde{u} \tilde{v}}\rangle=\left\langle\overline{u^{\prime} v^{\prime}}\right\rangle$ (where the prime refers to temporal variations about the temporal average, the overbar refers to a temporal average, and $<>$ refers to spatial average) and that $\left\langle\overline{u^{\prime} v^{\prime}}\right\rangle$ could be modeled as $\varepsilon_{\mathrm{M}}=\lambda \mathrm{d}_{\mathrm{h}} \mathrm{U}$ where $\left\langle\overline{\mathrm{u}^{\prime} \mathrm{v}^{\prime}}\right\rangle=-\varepsilon_{\mathrm{M}} \frac{\partial \mathrm{U}}{\partial \mathrm{r}}$ and $\lambda \cong 0.02$, and where $d_{h}=$ the hydraulic diameter, $u=$ the in-matrix average velocity, and $U=\langle u\rangle$ is the superficial, Darcian, or approach velocity. Therefore, for use in equation (6), and in the momentum equation, (2):

$$
\frac{\overline{\langle\tilde{u} \tilde{v}\rangle}}{\beta^{2}}=\frac{\overline{\langle\tilde{v} \tilde{u}\rangle}}{\beta^{2}} \approx \frac{\left\langle\overline{u^{\prime} v^{\prime}}\right\rangle}{\beta^{2}}=-\frac{1}{\beta^{2}} \varepsilon_{M} \frac{\partial U}{\partial r}=-\frac{1}{\beta^{2}} 0.02 d_{h} U \frac{\partial U}{\partial r}=-\frac{1}{\beta^{2}} \lambda d_{h} U \frac{\partial U}{\partial r}
$$




\section{Permeability and Inertial Coefficient}

In the fluid momentum equation, (2), the permeability, $K$, and the inertial coefficient, $C_{f}$, need evaluation for each type of porous medium. These coefficients can be evaluated for particular types of Stirling engine regenerator porous media via use of the friction-factor data from the oscillating-flow test rig given in Gedeon ${ }^{9}$ under the assumption that the flow is quasi-steady ${ }^{\dagger+t+19}$. The Darcy-Forchheimer steady-flow form of the 1-D fluid momentum equation, and a similar pressure-drop equation, but in terms of the Darcy friction factor, can be written, respectively:

$$
\begin{gathered}
\frac{\nabla p}{L}=\frac{\mu}{K} u+\frac{C_{f}}{\sqrt{K}}\langle\rho\rangle^{f} u^{2} \\
\frac{\nabla p}{L}=\frac{f_{D}}{d_{h}} \frac{1}{2}\langle\rho\rangle^{f} u^{2}
\end{gathered}
$$

From the oscillating-flow test rig data, as summarized by correlations given in the Sage manuals ${ }^{9}$, it was determined that friction-factor correlations for random fiber and wire screen are of the form:

$$
f_{D}=\frac{\alpha}{\operatorname{Re}}+\delta \operatorname{Re}^{\gamma} \text { where Reynolds } \#, \operatorname{Re}=\frac{\rho_{f} u d_{h}}{\mu}
$$

And where, for random fiber, $\alpha=192, \delta=4.53, \gamma=-0.067$, and for woven screen, $\alpha=129, \delta=2.91, \gamma=-0.103$. Substituting the expression for friction-factor and the definition of Reynolds number from equations (10), into equation (9), and then equating the right-hand sides of equations (8) and (9), it can be determined that:

$$
\frac{K}{d_{h}^{2}}=\frac{2}{\alpha} \quad \text { and } \quad C_{f}=\frac{\delta \operatorname{Re}^{\gamma}}{\sqrt{2 \alpha}}
$$

A frequently used expression for hydraulic diameter of random fiber and wire screen, in terms of porosity and wire diameter is:

$$
d_{h}=\frac{\beta}{1-\beta} d_{w}
$$

For the welded stacked-screens used in the UMN regenerator test module ${ }^{6}$ where $\beta=0.9$ and $d_{w}=0.81 \mathrm{~mm}$ or 8.1E-4 m, then $d_{h}=7.29 \mathrm{E}-3 \mathrm{~m}$. If it is also assumed that Reynolds No.'s are in the range from 25-100, as expected in the TDC regenerators, then equations (11) can be used to calculate values of permeability and inertial coefficient for TDC random fiber and the large screens used in the test module. The resulting TDC random fiber values are given in Table 1. The TDC random fiber results given in Table 1, in the rightmost column, are based on unidirectional flow tests of the entire TDC heater head (including heater, regenerator and cooler), taken from Wilson $^{19}$ The "UMN old" and "UMN new" values of permeability, in Table 1, are experimentally determined values of permeability and inertial coefficient, determined at an earlier and a later time ${ }^{\ddagger \neq \ddagger}$. The "CSU Calcs." are calculated values determined via microscopic CFD modeling of the UMN steady-flow test module ${ }^{\S \S \S \S}$. For the large scale-screens (see Table 1), results derived from the Sage empirical correlations and the microscopic CFD calculations are seen to be in relatively close agreement. The substantially greater differences of these values from the UMN test results have not been explained. The significance of differences such as these should be examined via a sensitivity study using a macroscopic porous-media model; this has not yet been done.

\section{Effective Fluid and Solid Thermal Conductivities}

In the fluid energy equation, (3), $\overline{\bar{k}}_{f e} \equiv$ an effective fluid conductivity tensor, each element of which is, in general, a sum of components due to molecular conductivity, thermal tortuosity conductivity and thermal dispersion conductivity. It can be broken down into these components as follows: From equation (3),

$$
\overline{\bar{k}}_{f e} \bullet \nabla\langle T\rangle^{f}=\overline{\bar{k}}_{f} \bullet \nabla\langle T\rangle^{f}+\frac{1}{V_{f}} \int_{A s f} \overline{\bar{k}}_{f} T d A-\rho_{f} C_{p}\langle\tilde{T} \tilde{\mathbf{u}}\rangle
$$

${ }^{+1+}$ Tew, R. C., Private Communication, 2005.

\#\#\# Simon, T., University of Minnesota, Private Communication, 2003.

§§§§ Ibrahim, M. B., Cleveland State University, Private Communication, 2003. 
Table 1. Values of Permeability, $\mathrm{K}$, and Inertial Coefficient, $\mathrm{C}_{\mathrm{f}}$, for the Large-Scale UMN Wire Screen and TDC Random Fiber Regenerator Materials.

\begin{tabular}{|c|c|c|c|c|c|c|}
\hline \multirow[t]{2}{*}{ Coefficient } & \multicolumn{4}{|c|}{$\begin{array}{l}\text { UMN Large-Scale Screens } \\
\left(d_{w}=8.1 E-4 \mathrm{~m}\right)\end{array}$} & \multicolumn{2}{|c|}{ TDC Random Fiber } \\
\hline & $\begin{array}{l}\text { UMN Old, } \\
\text { Experimental }\end{array}$ & $\begin{array}{l}\text { UMN New, } \\
\text { Experimental }\end{array}$ & CSU Calcs. & Sage Cor. & Sage Cor. & $\begin{array}{l}\text { Unidirectional } \\
\text { Flow Tests }\end{array}$ \\
\hline $\mathbf{K}\left(\mathbf{m}^{2}\right)$ & $1.07 \mathrm{E}-7$ & $1.86 \mathrm{E}-7$ & 8.9E-7 & $8.24 \mathrm{E}-7$ & $4.08 \mathrm{E}-10$ & $3.52 \mathrm{E}-10$ \\
\hline $\mathbf{K} / \mathbf{d}_{\mathbf{w}}{ }^{2}$ & 0.163 & 0.283 & 1.36 & 1.26 & - & - \\
\hline $\mathrm{C}_{\mathrm{f}}$ & 0.049 & 0.052 & 0.14 & $\begin{array}{c}0.13-0.11 \\
\operatorname{Re}=25-100\end{array}$ & $\begin{array}{c}0.19-0.17 \\
\operatorname{Re}=25-100\end{array}$ & $\begin{array}{c}0.154-0.095 \\
R e=25-100\end{array}$ \\
\hline
\end{tabular}

where:

$$
\frac{1}{V_{f}} \int_{A s f} \overline{\bar{k}}_{f} T d A \equiv \overline{\bar{k}}_{t o r} \bullet \nabla\langle T\rangle^{f}
$$

Equation (14) defines the thermal tortuosity thermal conductivity, $\overline{\bar{k}}_{t o r}$.

$$
-\rho_{f} C_{p}\langle\tilde{T} \tilde{\mathbf{u}}\rangle \equiv \overline{\bar{k}}_{d i s} \cdot \nabla\langle T\rangle^{f}
$$

Equation (15) defines the thermal dispersion thermal conductivity, $\overline{\bar{k}}_{d i s}$.

$$
\therefore \quad \overline{\bar{k}}_{f e} \bullet \nabla\langle T\rangle^{f}=\overline{\bar{k}}_{f} \bullet \nabla\langle T\rangle^{f}+\overline{\bar{k}}_{\text {tor }} \bullet \nabla\langle T\rangle^{f}+\overline{\bar{k}}_{d i s} \bullet \nabla\langle T\rangle^{f}
$$

Equation (16) defines the effective fluid thermal conductivity, $\overline{\bar{k}}_{f e}$.

$$
\text { and } \quad \overline{\bar{k}}_{f e}=\overline{\bar{k}}_{f}+\overline{\bar{k}}_{f, \text { tor }}+\overline{\bar{k}}_{d i s}=\overline{\bar{k}}_{f, \text { stag }}+\overline{\bar{k}}_{d i s}
$$

Where, in the above equation, the sum of the fluid molecular and thermal tortuosity conductivities are lumped together and called the fluid stagnant thermal conductivity.

It is assumed that only the diagonal elements of the effective fluid conductivity tensor are non-zero. Then in terms of 3-D cylindrical coordinates which are appropriate for Stirling engine simulation, it is further assumed that:

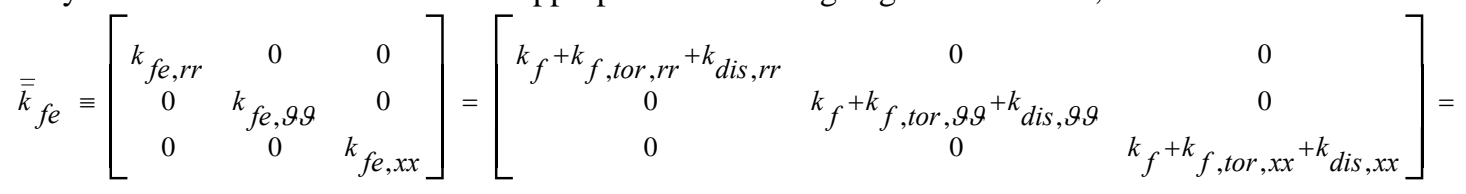

$$
\left[\begin{array}{ccc}
k_{f, s t a g, r r}+k_{d i s, r r} & 0 & 0 \\
0 & k_{f, s t a g, \vartheta \vartheta^{+k}}{ }_{d i s, \vartheta \vartheta} & 0 \\
0 & 0 & k_{f, s t a g, x x}+k_{d i s, x x}
\end{array}\right]
$$

In the above tensor equation for fluid effective thermal conductivity, (18), molecular conductivity is isotropic, and fluid tortuosity and dispersion conductivities will be assumed anisotropic. In general, the tortuosity conductivity is different in different directions when the alternating fluid/solid geometry is different when looking in different directions. Therefore, when fluid molecular and thermal tortuosity conductivities are lumped together to form the stagnant thermal conductivity, the stagnant thermal conductivity is also, in general, different in different directions - as dictated by the geometry of the matrix. The thermal dispersion conductivity has contributions from an advective term and an eddy term. In the flow direction there would be both. Normal to the flow direction, there is only the eddy dispersion term.

In the solid energy equation, (4), the effective solid conductivity, $\overline{\bar{k}}_{s e}$, is defined: 


$$
\overline{\bar{k}}_{s e} \equiv\left[\begin{array}{ccc}
k_{s e, r r} & 0 & 0 \\
0 & k_{s e, \vartheta \vartheta} & 0 \\
0 & 0 & k_{s e, x x}
\end{array}\right]=\left[\begin{array}{ccc}
f\left(k_{s}, k_{s, t o r, r r}\right) & 0 & 0 \\
0 & f\left(k_{s}, k_{s, t o r, \vartheta \vartheta}\right) & 0 \\
0 & 0 & f\left(k_{s}, k_{s, \text { tor }, x x}\right)
\end{array}\right]
$$

The molecular thermal conductivities can be assumed to be well known. That leaves to be determined: (1) fluid thermal dispersion and (2) fluid stagnant conductivities and solid effective conductivities-which are each functions of the molecular conductivity and the thermal tortuosity conductivity, for the fluid or solid; thus the form of the last matrix of equation (19).

\section{Thermal Dispersion Conductivity}

$\mathrm{Niu}^{7}$ and other researchers, have measured eddy diffusivity (or transport) in, or very close to, porous media. If it is assumed that this is also equivalent to thermal dispersion due to eddies, as in Niu ${ }^{7}$, based on the Reynolds Analogy, then values for thermal dispersion due to eddies are given in Table 2, below, in terms of porous media hydraulic diameter, $d_{h}$, and superficial, approach, or Darcian velocity, $U=<u>$. Although in general thermal dispersion by eddies is expected to be anisotropic, for this initial model it may be adequate to use the same relationship to calculate eddy dispersion in all directions. It should be noted, however, that in the flow direction, total dispersion is by eddies and by advection, where the advection term is dominant. For a direction normal to the flow, dispersion is only by eddies. To explore further possible differences of eddy dispersion in different directions refer to $\mathrm{Niu}^{6-8}$, McFadden ${ }^{20}$ and Simon ${ }^{21}$. Once a macroscopic thermal non-equilibrium model is implemented in a Stirling CFD code, the significance of the differences in Table 2 can be explored via a sensitivity study.

Table 2. A Comparison of Thermal Dispersion Coefficients from Several Methods. Taken from Niu'

\begin{tabular}{|l|c|c|}
\hline & Estimated Thermal Dispersion & Porous media \\
\hline $\begin{array}{l}\text { Current Direct } \\
\text { Measurements at UMN, } \\
\text { Niu }\end{array}$ & $\varepsilon_{M, e d d y}=\frac{k_{d i s, y y}}{\rho_{f} c_{p}}=0.02 d_{h} U \quad$ or $\quad \frac{k_{d i s, y y}}{k_{f}}=0.02 P e$ & Welded Screen \\
\hline Hunt and Tien & \multicolumn{1}{|c|}{$\frac{k_{d i s, y y}}{k_{f}}=0.0011 P e$} & Fibrous Media \\
\hline $\begin{array}{l}\text { Metzger, Didierjean, } \\
\text { and Maillet }^{23}\end{array}$ & $\frac{k_{d i s, y y}}{k_{f}}=(0.03-0.05) P e$ and $\frac{k_{d i s, x x}}{k_{f}}=0.073 P e^{1.59}$ & Packed Spheres \\
\hline Gedeon $^{9}$ & $\frac{k_{d i s, x x}}{k_{f}}=0.50 P e^{0.62} \beta^{-2.91}$ or $\frac{k_{d i s, x x}}{k_{f}} \approx 0.06 P e$ for $\beta=0.9, P e=560$ & Woven Screen \\
\hline
\end{tabular}

\section{Fluid-Stagnant and Solid-Effective Thermal Conductivity}

The fluid-stagnant and solid-effective thermal conductivities (each a function of the appropriate molecular and thermal tortuosity conductivities) are estimated based on the geometry of the matrix of interest. McFadden ${ }^{20}$ calculated a radial stagnant conductivity for the welded screens based on considerations of the geometry of the large-scale wire screens. Similar calculations are made for a fluid-saturated metal foam in Boomsma ${ }^{24}$. In the case of the Stirling TDC that has been modeled with CFD codes, a random fiber matrix is used in the regenerator. Since the details of the geometry for such a matrix are random, some assumptions must be made, as discussed below.

For comparison with the calculations of McFadden $^{20}$, mentioned above, the same molecular thermal conductivities used by McFadden will be used here (i.e. for air and stainless steel): So, assume that: $k_{s}=13.4 \mathrm{~W} / \mathrm{m}-$ K for Stainless Steel 316, $k_{f}=0.026 \mathrm{~W} / \mathrm{m}-\mathrm{K}$ for air at standard temperature, and $\beta=0.90$.

In the random fiber matrix most of the lengths of the fibers are believed to lie in planes perpendicular to the main flow axis. Therefore, initially make the assumption that for a 3-D CFD model, the effective solid+fluid conductivity in the radial and azimuthal directions (that would be appropriate for use in an equilibrium porous-media model) follows the parallel model defined below, and the effective solid+fluid conductivity in the axial direction follows the series model, also defined below.

The parallel model for this lumped effective conductivity for fluid and solid, assuming all of the fibers run in the same direction (not including the fluid thermal dispersion) is: 


$$
k_{e f f, s+f}=k_{f} \beta+k_{s}(1-\beta)
$$

Therefore, this effective solid+fluid conductivity for air and stainless steel combined, for a 90\% porosity matrix, would be:

$$
\begin{aligned}
k_{\text {eff }, s+f} & =\left(26 \times 10^{-3} \mathrm{~W} / \mathrm{mK}\right)(0.90)+(13.4 \mathrm{~W} / \mathrm{mK})(0.1) \\
& =0.0234 \mathrm{~W} / \mathrm{mK}+1.34 \mathrm{~W} / \mathrm{mK}=1.36 \mathrm{~W} / \mathrm{mK}
\end{aligned}
$$

As already mentioned, the above would be an appropriate effective solid+fluid thermal conductivity for an equilibrium porous media model. However for a non-equilibrium porous media model, assume that the two terms on the right of the above equation represent the fluid stagnant thermal conductivity and the solid effective conductivity, respectively. That is, assume:

$$
\begin{gathered}
k_{f, \text { stag }}=k_{f} \beta=\left(26 \times 10^{-3} \mathrm{~W} / \mathrm{mK}\right)(0.90)=0.0234 \mathrm{~W} / \mathrm{mK} \\
k_{s e}=k_{s}(1-\beta)=(13.4 \mathrm{~W} / \mathrm{mK})(0.1)=1.34 \mathrm{~W} / \mathrm{mK}
\end{gathered}
$$

However, in accordance with McFadden ${ }^{20}$ :

$$
k_{s} / k_{f}=13.4 / 26 \times 10^{-3}=515 \text { and } k_{\text {eff }, s+f} / k_{f}=1.36 / 26 \times 10^{-3}=52
$$

The value 52 is considerably larger than the 32.5 estimated by UMN for their wire screen in McFadden ${ }^{20}$, based on average geometrical considerations, rather than the parallel model. Since wire screen and random fiber are thought to have similar heat transfer properties, perhaps the solid part of the parallel model should be corrected to the UMN wire screen value by multiplication by the correction factor:

$$
\text { kcorrect }=32.5 / 52=0.625
$$

Then the corrected parallel values for use for random fiber in the radial and azimuthal directions would be:

$$
\begin{gathered}
k_{\text {eff }, s+f}=0.0234+1.36 \times 0.625=0.873 \quad \text { (for equil., model) } \\
k_{s e}=k_{s}(1-\beta)_{0.625}=1.34 \mathrm{~W} / \mathrm{mK} \times 0.625=0.838 \quad \text { (for } \text { non }- \text { equil. model, radial \& azimuthal directions) }
\end{gathered}
$$

For the axial direction, the lumped effective solid + fluid effective conductivity, $k_{e f f, s+f}$, (which also doesn’t include thermal dispersion) should be substantially less than in the radial and azimuthal directions. Initially assume the series model mentioned in Ref. 14. That is:

$$
k_{\text {eff }, s+f}=\left(\frac{1}{\frac{\beta}{k_{f}}+\frac{1-\beta}{k_{s}}}\right)=\left(\frac{1}{\frac{0.90}{26 \times 10^{-3}}+\frac{0.1}{13.4}}\right)=0.0289 \mathrm{~W} / \mathrm{mK}
$$

This axial effective solid+fluid thermal conductivity (not including thermal dispersion) is just slightly larger than the molecular fluid conductivity for air of $0.026 \mathrm{~W} / \mathrm{m}-\mathrm{K}$ and is probably too small since this series model assumes that the wires are not touching in the axial direction. 3-D CFD microscopic simulations of a representative elementary volume of the UMN regenerator by Rong ${ }^{* * * * *}$, suggest that the value shown in equation (21) should be increased by a factor of 2.157. Therefore assume that:

$$
k_{\text {eff }, s+f}=\left(\frac{2.157}{\frac{\beta}{k_{f}}+\frac{1-\beta}{k_{s}}}\right)=\left(\frac{2.157}{\frac{0.90}{26 \times 10^{-3}}+\frac{0.1}{13.4}}\right)=(0.0289 \mathrm{~W} / \mathrm{mK})(2.157)=0.0623 \mathrm{~W} / \mathrm{mK} \text { (axial direction) }
$$

This resulting effective solid+fluid thermal conductivity in the axial direction would be appropriate for an equilibrium model. Seeing no obvious way to separate values for fluid and solid for a non-equilibrium macroscopic porous media model, based on the series model, we propose initially using this same value for both the fluidstagnant and solid-effective conductivities in the axial direction in the non-equilibrium model, also-hoping that the overall axial effect might be reasonable.

Recall that to get the total effective fluid conductivities in different directions, the thermal dispersion conductivity should be added to the fluid stagnant conductivities in the radial, azimuthal and axial directions. Also, for use in modeling the TDC regenerator, the above effective conductivities must be recalculated using the thermal conductivity of helium instead of air.

${ }^{* * * * *}$ Rong, W., Cleveland State University, Private Communication, 2005. 
Literature searches for experimental effective fluid+solid effective thermal conductivities (appropriate for an equilibrium porous-media model) might prove useful. One attempt to locate such information via the website of the National Institute of Standards and Technology (NIST) was not successful.

\section{Heat Transfer Coefficient between Fluid and Solid Matrix Elements}

A good source of heat transfer correlations for wire screen and random fiber Stirling regenerator materials is Gedeon ${ }^{9}$. These correlations are based on experimental data from the NASA/Sunpower oscillating-flow test rig; this rig was designed and fabricated specifically for the purpose of determining friction-factor and heat-transfer correlations for use in Stirling device design and modeling. Heat transfer correlations in terms of Nusselt No., Peclet Number (Reynolds No. x Prandtl No.), and porosity are:

For wire screen, $N u=\left(1 .+0.99 P e^{0.66}\right) \beta^{1.79}$ and for random fiber, $N u=\left(1 .+1.16 P e^{0.66}\right) \beta^{2.61}$ where

$$
N u=\frac{h d}{k}, P e=\operatorname{Re} \operatorname{Pr}=\frac{\rho u d_{h}{ }^{c} p^{\mu}}{\mu}
$$

Measurements by $\mathrm{Niu}^{25}$ indicated that the above correlation and its application in a quasi-steady fashion is suitable for the portion of an oscillatory cycle when the acceleration is sufficiently weak or the flow is decelerating, but during strong acceleration, the unsteady measurements indicate a violation of the quasi-steady-flow assumption characterized by a lag between the heat flux from one phase of the matrix to the other and the temperature difference between phases. The measurements also showed an apparently poor mixing of the flow in the pore during the strong acceleration portions of the cycle. The Valensi number based on molecular (rather than eddy) viscosity) of the flow was 2.1, which is a bit higher than values in the general operating range of an engine regenerator $(\sim 0.23$ for one engine). Thus, this unsteady effect may have been overestimated. The data also indicate that over the full cycle, an estimate given by the correlation above and applied as if the flow were quasi-steady is a reasonable approach for this initial or "draft" model.

\section{Concluding Remarks}

A set of transient, compressible-flow, conservation equations is summarized for reference in defining the parameters whose values are needed for a macroscopic, thermal-non-equilibrium porous-media model. Such a porous-media model is needed in existing commercial CFD codes (such as CFD-ACE and Fluent) in order to more accurately model the regenerator heat exchanger in Stirling engine devices (since only equilibrium porous-media models are now available in the Fluent and CFD-ACE codes). Available experimental information from large-scale wire-screen testing is used to define a hydrodynamic-dispersion term in the momentum equation. Experimental information is also used for definition of the permeability and inertial coefficients in the momentum equation and for the thermal-dispersion conductivity for the regenerator fluid. Methods are also outlined for estimating the stagnantfluid and effective-solid thermal conductivities. Thus adequate information is presented for definition of an initial, or "draft," non-equilibrium porous media model for use in CFD regenerator modeling of Stirling devices. It is anticipated that use of this initial model in CFD codes may demonstrate that further work on refinement of the nonequilibrium porous-media model and its parameters will be needed.

\section{References}

${ }^{1}$ Schreiber, J. G. and Thieme, L.G., “Accomplishments of the NASA GRC Stirling Technology Development Project,” in proceedings of $2^{\text {nd }}$ International Energy Conversion Engineering Conference, AIAA-2004-5517, Providence, RI, 2004.

${ }^{2}$ Thieme, L. G. and Schreiber, J. G., “Advanced Technology Development for Stirling Convertors,” in proceedings of Space Technology and Applications International Forum (STAIF 2004), edited by M. El-Genk, AIP Conference Proceedings 654, Melville, New York, pp. 432-439, 2004.

${ }^{3}$ Dyson, R. W., Wilson, S. D., and Demko, R., “On the Need for Multidimensional Stirling Simulations,” in proceedings of

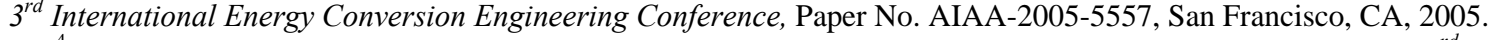

${ }^{4}$ Dyson, R. W., Wilson, S. D., and Demko, R., "Fast Whole-Engine Stirling Analysis," in proceedings of $3^{\text {rd }}$ International Energy Conversion Engineering Conference, Paper No. AIAA-2005-5558, San Francisco, CA, 2005.

${ }^{5}$ Ibrahim, M., Mittal, M., Jiang, N., and Simon, T., "Validation of Multi-Dimensional Stirling Engine Codes: Modeling of the Heater Head," in proceedings of $3^{\text {rd }}$ International Energy Conversion Engineering Conference, Paper No. AIAA-2005-5654, San Francisco, CA, 2005.

${ }^{6}$ Niu, Y., Simon, T.W., Ibrahim, M.B., Tew, R., and Gedeon, D., “JET Penetration into a Stirling Engine Regenerator Matrix with Various Regenerator-to-Cooler Spacings,” in proceedings of 1st International Energy Conversion Engineering Conference, Paper No. AIAA-2003-6014, Portsmouth, VA, 2003. 
${ }^{7}$ Niu, Y., Simon, T., Gedeon, D. and Ibrahim, M., “On Experimental Evaluation of Eddy Transport and Thermal Dispersion in Stirling Regenerators," in proceedings of $2^{\text {nd }}$ International Energy Conversion Engineering Conference, Paper No. AIAA2004-5646, Providence, RI, 2004.

${ }^{8}$ Niu, Y., McFadden, G., Simon, T., Ibrahim, M. and Wei, R., "Measurements and Computation of Thermal Dispersion in a Porous Medium,” in proceedings of $3^{\text {rd }}$ International Energy Conversion Engineering Conference, Paper No. AIAA-2005-5578, San Francisco, CA, 2005.

${ }^{9}$ Gedeon, D., “Sage Stirling Cycle Model Class Reference Guide,” $3^{\text {rd }}$ Edition, Gedeon Associates, Athens, OH, 1999.

${ }^{10}$ Ibrahim, M. B., Rong, W., Simon, T., Tew, R., and Gedeon, D., "Simulations of Flow and Heat Transfer inside Regenerators Made of Stacked Welded Screens Using Periodic Cell Structures,” in proceedings of $2^{\text {nd }}$ International Energy Conversion Engineering Conference, Paper No. AIAA-2004-5599, Providence, RI, 2004.

${ }^{11}$ Singh, C., Tathgir, R. G., and Muralidhar, K., "Comparison of 1-equation and 2-equation Models for Convective Heat Transfer in Saturated Porous Media,” The Institution of Engineers (India) Journal, Vol. 84, MC 3, pp. 104-113, October 2003.

${ }^{12}$ Amiri, A. and Vafai, K., “Transient Analysis of Incompressible Flow through a Packed Bed," Int. J. Heat Mass Transfer, Vol. 41, pp. 4259-4279, 1998.

${ }^{13}$ Minkowcyz, W. J., Haji-Sheikh, A. and Vafai, K., “On Departure from Local Thermal Equilibrium in Porous Media due to a Rapidly Changine Heat Source: The Sparrow Number,” Int. J. Heat Mass Transfer, Vol. 42, pp. 3373-3385, 1999.

${ }^{14}$ Muralidhar, K. and Suzuki, K., “Analysis of Flow and Heat Transfer in a Regenerator Mesh using a Non-Darcy Thermally Non-Equilibrium Model,” Int. J. Heat Mass Transfer, Vol. 44, pp. 2493-2504, 2001

${ }^{15}$ Gedeon, D., “Mainifest: A Computer Program for 2-D Flow Modeling in Stirling Machines,” NASA CR 182290, 1989.

${ }^{16}$ Whitaker, S., “The Forchheimer Equation: A Theoretical Development,” Transport in Porous Media, Vol. 25, pp.27-61, 1996.

${ }^{17}$ Burmeister, L. C., Convective Heat Transfer, $2^{\text {nd }}$ Ed., pp 48-50, Wiley, 1993.

${ }^{18}$ Kaviany, M., Principles of Heat Transfer in Porous Media, Spring-Verlag, Mechanical Engineering Series, $2^{\text {nd }}$ Edition, pp 66-67 and 47, 1995.

${ }^{19}$ Wilson, S. D., Dyson, R. W., Tew, R. C., and Demko, R., "Experimental and Computational Analysis of Unidirectional Flow through Stirling Engine Heater Head,” $3^{\text {rd }}$ International Energy Conversion Engineering Conference, Paper No. AIAA2005-5539, San Francisco, CA, 2005.

${ }^{20}$ McFadden, G., “Forced Thermal Dispersion Within a Representative Stirling Engine Regenerator," University of Minnesota Master's Thesis, 2005.

${ }^{21}$ Simon, T., McFadden, G. and Ibrahim, M., “Thermal Dispersion within a Porous Medium Near a Solid Wall," To be Presented at International Heat Transfer Conference, Sydney, Australia, Aug. 13-18, 2006.

${ }^{22}$ Hunt, M. L. and Tien, C. L., "Effects of Thermal Dispersion on Forced Convection in Fibrous Media," Int. J. Heat Mass Transfer, Vol. 31, pp. 301-308, 1988.

${ }^{23}$ Metzger, T., Didierjean, S. and Maillet, D., “Optimal Experimental Estimation of Thermal Dispersion Coefficients in Porous Media,” Int. J. Heat Mass Transfer, Vol. 47, pp. 3341-3353, 2004.

${ }^{24}$ Boomsma, K., and Poulikakos, D., "On the Effective Thermal Conductivity of a Three-Dimensionally Structured FluidSaturated Metal Foam,” Int. J. of Heat \& Mass Transfer, Vol. 44, pp. 827-836, 2001.

${ }^{25}$ Niu, Y., Simon, T.W., Ibrahim, M.B., Tew, R., and Gedeon, D., "Measurements of Unsteady Convective Heat Transfer Rates within a Stirling Regenerator Matrix Subjected to Oscillatory Flow," in proceedings of 1st International Energy Conversion Engineering Conference, Paper No. AIAA-2003-6013, Portsmouth, VA, 2003.

${ }^{26}$ Bird, R. B., Steward, W. E., and Lightfoot, E. N., Transport Phenomena, 1st Ed., Chapter 3 for Continuity and Momentum Equations, Chapter 10 for Fluid Energy Equation, Wiley, 1960. 
Public reporting burden for this collection of information is estimated to average 1 hour per response, including the time for reviewing instructions, searching existing data sources, gathering and maintaining the data needed, and completing and reviewing the collection of information. Send comments regarding this burden estimate or any other aspect of this Davis Highway, Suite 1204, Arlingtongestions for reducing this burden, to Washington Headquarters Services, Directorate for Information Operations and Reports, 1215 Je

\begin{tabular}{|l|l|l|}
\hline 1. AGENCY USE ONLY (Leave blank) & $\begin{array}{c}\text { 2. REPORT DATE } \\
\text { October } 2006\end{array}$ & $\begin{array}{r}\text { 3. REPORT TYPE AND DATES COVERED } \\
\text { Technical Memorandum }\end{array}$
\end{tabular}

4. TITLE AND SUBTITLE 5. FUNDING NUMBERS

An Initial Non-Equilibrium Porous-Media Model for CFD Simulation of Stirling Regenerators

6. AUTHOR(S)

WBS 138494.04.01.01

Roy C. Tew, Terry Simon, David Gedeon, Mounir Ibrahim, and Wei Rong

7. PERFORMING ORGANIZATION NAME(S) AND ADDRESS(ES)

National Aeronautics and Space Administration

John H. Glenn Research Center at Lewis Field

Cleveland, Ohio 44135-3191

8. PERFORMING ORGANIZATION REPORT NUMBER

E-15669

9. SPONSORING/MONITORING AGENCY NAME(S) AND ADDRESS(ES)

10. SPONSORING/MONITORING AGENCY REPORT NUMBER

National Aeronautics and Space Administration

Washington, DC 20546-0001

NASA TM-2006-214391

\section{SUPPLEMENTARY NOTES}

Prepared for the Fourth International Energy Conversion Engineering Conference and Exhibit (IECEC) sponsored by the American Institute of Aeronautics and Astronautics, San Diego, California, June 26-29, 2006. Roy C. Tew, NASA Glenn Research Center; Terry Simon, Department of Mechanical Engineering, University of Minnesota, 111 Church Street, S.E., Minneapolis, Minnesota 55455; David Gedeon, Gedeon Associates, 16922 South Canaan Road, Athens, Ohio 45701; Mounir Ibrahim and Wei Rong, Department of Mechanical Engineering, Cleveland State University, 1960 East 24th Street, Cleveland, Ohio 44115. Responsible person, Roy C. Tew, NASA Glenn Research Center, organization code RPT, 216-433-8471.

\begin{tabular}{l|l} 
12a. DISTRIBUTION/AVAILABILITY STATEMENT & 12b. DISTRIBUTION CODE
\end{tabular}

Unclassified - Unlimited

Subject Category: 20

Available electronically at http://gltrs.grc.nasa.gov

This publication is available from the NASA Center for AeroSpace Information, 301-621-0390.

13. ABSTRACT (Maximum 200 words)

The objective of this paper is to define empirical parameters for an initial thermal non-equilibrium porous-media model for use in Computational Fluid Dynamics (CFD) codes for simulation of Stirling regenerators. The two codes currently used at Glenn Research Center for Stirling modeling are Fluent and CFD-ACE. The codes' porous-media models are equilibrium models, which assume solid matrix and fluid are in thermal equilibrium. This is believed to be a poor assumption for Stirling regenerators; Stirling 1-D regenerator models, used in Stirling design, use non-equilibrium regenerator models and suggest regenerator matrix and gas average temperatures can differ by several degrees at a given axial location and time during the cycle. Experimentally based information was used to define: hydrodynamic dispersion, permeability, inertial coefficient, fluid effective thermal conductivity, and fluid-solid heat transfer coefficient. Solid effective thermal conductivity was also estimated. Determination of model parameters was based on planned use in a CFD model of Infinia's Stirling Technology Demonstration Converter (TDC), which uses a random-fiber regenerator matrix. Emphasis is on use of available data to define empirical parameters needed in a thermal non-equilibrium porousmedia model for Stirling regenerator simulation. Such a model has not yet been implemented by the authors or their associates.

14. SUBJECT TERMS

Stirling engines; Porous media model; Regenerator; Computerized simulation; CFD

\begin{tabular}{|c|c|c|}
\hline $\begin{array}{c}\text { 17. SECURITY CLASSIFICATION } \\
\text { OF REPORT } \\
\text { Unclassified }\end{array}$ & $\begin{array}{c}\text { 18. SECURITY CLASSIFICATION } \\
\text { OF THIS PAGE } \\
\text { Unclassified }\end{array}$ & $\begin{array}{c}\text { 19. SECURITY CLASSIFICATION } \\
\text { OF ABSTRACT } \\
\text { Unclassified }\end{array}$ \\
\hline
\end{tabular}

NSN 7540-01-280-5500

Standard Form 298 (Rev. 2-89) 

